\begin{tabular}{|l|c|}
\hline & $\begin{array}{c}\text { Biological Sciences } \\
\text { (An International Journal of Medical, Pharmaceutical, and Life Sciences) } \\
\text { Research }\end{array}$ \\
Publication
\end{tabular}

\title{
Nose-to-Brain drug delivery via nanocarriers for the management of neurodegenerative disorders: recent advances and future
}

\author{
Vishesh Singh*, Arun Singh Lalotra, Shelly Agrawal, Gaurav Mishra \\ Department of Pharmaceutics, ISF College of Pharmacy, Moga, Punjab, 142001, India
}

\section{ARTICLEHISTORY}

Received: 07-04-2021

Revised: 08-04-2021

Accepted: 09-04-2021

Online: 09-04-2021

\section{KEYWORDS}

Neurodegenerative disorders

Nasal targeting

Nanocarriers

Nanoparticles

Micelles

Liposomes

Nano-emulsion

\section{ABSTRACT}

Neurodegenerative disorders like Parkinson's disease, Huntington's disease, Amyotrophic lateral sclerosis, Alzheimer's disease, and meningitis are caused by a decline in neuronal synaptic function, which leads to neuronal cell death. Oral administration of drugs for neurodegenerative disorders results in low oral bioavailability due to its systemic absorption. The bloodbrain barrier allows the passage of only lipophilic drugs having a size less than $200 \mathrm{~nm}$. Various drugs get metabolized before reaching the $\mathrm{BBB}$ resulting in the low drug concentration in the brain. Nose-to-brain delivery is a simple and direct way of brain targeting as it bypasses BBB and hepatic-first pass metabolism. The mechanism of action of nanocarrier delivery in the brain is via olfactory and trigeminal nerves. Several studies reported the effective use of nanocarriers for neurodegenerative disorders. The nanocarriers can be either lipid-based or polymer-based. The drawbacks associated with the conventional oral treatment of neurodegenerative disorders can be overcome by direct nose-tobrain delivery. In the present review, we have discussed several physiological barriers, including the BBB on drug therapy. Further, we have discussed novel brain targeting strategies via a nasal route that provides better therapeutic effects.

\section{Introduction}

Neurodegenerative disorders like Parkinson's

*Address for correspondence

Department of Pharmaceutics, ISF College of

Pharmacy, Moga, Punjab, India

Email: svishesh01@gmail.com

DOI: http://dx.doi.org/10.55006/biolsciences.2021.1103 Published by IR Research Publication; Singh V et al (C) 2021 by Biological Sciences is licensed under CC BY 4.0 (c) disease, Huntington's disease, Amyotrophic lateral sclerosis, Alzheimer's disease, etc., are caused by a decline in neuronal synaptic function, which leads to neuronal cell death. Neurodegenerative diseases are characterized by progressive loss of structure or function of neurons, including neurons' death. Degeneration can be found in many different levels of neuronal circuitry. Depending on the disease type or level, it ranges from molecular to a systemic level. Neurodegenerative diseases are of two forms- Hereditary form, sporadic form. Several neurodegenerative disorders cause postural imbalance and muscle stiffness. Orally administered drugs for the treatment of neurodegenerative disorders generally have low 
oral bioavailability due to hepatic first-pass metabolism and less systemic absorption. The drug enters into the systemic circulation and starts getting metabolizing and elimination before reaching the targeted organ, i.e., the brain, leading to a low concentration of the drug at the targeted site with respect to other non-targeted organs. Various barriers associated with brain drug delivery, such as the blood-brain barrier (BBB) and blood-cerebrospinal fluid barrier (BCSFB) play an important role in the diffusion of drugs into the brain treating neurological diseases. Due to its unique anatomical structure, BBB has a pivotal role in extricating the CNS from systemic circulation and limits the entry of molecules into the brain depending on their size and endothelial permeability. BCSFB also splits the blood from the cerebrospinal fluid. The BBB allows the passage of only lipophilic drugs having a size of less than 200 $\mathrm{nm}$. Nose-to-brain delivery is a simple and direct way of brain targeting as it bypasses BBB and hepatic-first pass metabolism. The mechanism of action of nanocarrier delivery in the brain is via olfactory and trigeminal nerves. On intranasal administration, the administered drug molecules come in contact with the nasal mucosa, which is innervated by olfactory nerves, trigeminal nerves. The only concern with nasal administration is the mucociliary clearance of the drug, which may lead to degradation of the drug before reaching the brain. Mucociliary clearance regulates the residence time of the drug in the nasal mucosa. Several studies reported the effective use of nanocarriers for neurodegenerative disorders. The nanocarriers can be either lipid-based or polymerbased. The drawbacks associated with the conventional oral treatment of neurodegenerative disorders can be overcome by direct nose-to-brain delivery. In the present review, we have discussed several physiological barriers, including the BBB on drug therapy. Further, we have discussed novel brain targeting strategies via a nasal route that provides better therapeutic effects. Some of the nanocarriers which have reported for brain targeting, such as nanoemulsion/microemulsion, polymeric nanoparticles, polymeric micelles, dendrimers, nanogel, liposomes, lipid nanoparticles, solid lipid nanoparticles, emulsomes, etc. are discussed in the present review as these showed better therapeutic effects in the treatment of neurodegenerative disorders.

\section{Neurodegenerative disorders}

Disorders that are associated with atrophy of the central nervous system (CNS) structures are termed as neurodegenerative disorders (NDs) [1]. Neurodegenerative disorders, like Parkinson's disease (PD), Huntington disease (HD), Amyotrophic lateral sclerosis (ALS), Alzheimer's disease (AD) and additionally meningitis are characterized with progressive loss of structure or function of neurons, including death of neurons. Degeneration can be found in many different levels of neuronal circuitry. Depending the disease type or level, it ranges from molecular to systemic level. The common key pathological features of neurodegenerative diseases include excessive generation of oxidative and nitrosative stress, accumulation of misfolded proteins (e.g., $\alpha$ synuclein and amyloid- $\beta[A \beta]$ ), the appearance of dysfunctional mitochondria, and increased synaptic damage [2]. Neurological diseases may exhibit distinct pathological and clinical manifestations among patients, but in general encompass a broad spectrum of pathological conditions, which result in alterations in neural function and progressive loss of neural tissue. Neurodegenerative diseases are of two forms- Hereditary form and sporadic form. Hereditary neurodegenerative diseases are often caused by genetic mutations that occur in rare diseases like cardiomyopathy and arrhythmia whereas the other sporadic form is caused by the production of excess reactive oxygen and nitrogen species (ROS/RNS), produced by exposure to unknown environmental risk factors [2].

Certain molecular pathways along with synapses, neuronal subpopulations and local circuits in specific brain regions, as well as higher-order neural networks are believed to be disturbed in neurodegenerative disorders. Abnormal network activities may also lead to the vicious cycle, which may further impair the integrity and functions of neurons and synapses [1].

ROS and RNS, reactive molecules, are implicated both in physiological and pathological processes of brain function that depend on the severity and length of the ROS/RNS-associated stress in the brain. These free radical species aberrantly increase the generation of nitric oxide $(\bullet N O)$ related species that lead to accelerating the manifestation of key neuropathological features of the disease [2].

\section{Types of neurodegenerative disorders}

\section{Alzheimer's disease}

It is a CNS disorder which is characterized by progressive deterioration of neurons that result in loss of cognitive behavior, memory impairment, and disturbance in a daily routine activity like brushing, bathing, eating, drinking, communication, reading, writing, etc. thus it overall 
disturbs the thinking ability and may also lead to mental illness [3]. Extracellular deposits of $A \beta$ peptide and intra-neuronal accumulations of tau protein in the brain, being the main histopathological features of AD [4].

\section{Parkinson's disease}

It is another chronic progressive neurodegenerative disorder that is usually characterized by the progressive loss of dopaminergic neurons in the substantia nigra, which has a prevalence rate of $1 \%$ amongpopulation of over 65 years of age [4].

\section{Amyotrophic lateral sclerosis}

It is an untreatable and fatal progressive degenerative disease involving motor neuron cell death [4]. Gradual degeneration of nerve cells of the central nervous system that control voluntary muscle movement indicates the occurrence of this disease. Degeneration of motor neurons is characterized by muscle weakness, typically impacting arms and legs, speech, swallowing and breathing [5].

\section{Huntington disease}

It is a genetic neurodegenerative disease, which is caused by abnormal trinucleotide repeat sequences (CAG) expansion [4]. This disease generally progresses with age and is characterized by the primary symptom, chorea, uncontrolled motor behavior [6].

\section{Conventional treatment of neurodegenerative disorders}

There are limited clinical treatments for neurodegenerative diseases that do not completely cure the disease but only slow down its progress. Various clinically available drugs for $A D$ are donepezil, rivastigmine, galantamine and memantine [7]. In the case of Parkinson's disease, Levodopa is used as first-line treatment, while other drugs such as selegiline, amantadine, ropinirole, rotigotine, etc are also used as second-line drugs, with L-DOPA still being the gold standard therapy for PD. But, as the disease progresses, it becomes less controllable and motor complications including fluctuations and levodopa-induced dyskinesia (LID), eventually develops [8]. However, oral administration of drugs for the treatment of neurodegenerative disorders usually results in low bioavailability due to hepatic first-pass metabolism that follows less drug concentration in brain vs. other organs. Hence, the drug will not produce optimum therapeutic effects as supposed to. Administered as an oral tablet and absorbed in the proximal small bowel, levodopa crosses the bloodbrain barrier via a large neutral amino acid active carrier system [9]. Oral levodopa formulations have been developed to provide more constant plasma levodopa concentrations [10]. Bioavailability and the therapeutic efficacy were found to be reduced by major metabolism of L-DOPA, through oxidation, decarboxylation, and O-methylation. The side effects associated with the metabolic products of L-DOPA are cardiac arrhythmias, vomiting, and nausea. Generally, around $95 \%$ of LDOPA is metabolized in the liver, plasma, and GIT, whereas only $1 \%$ of the dose of L-DOPA penetrates the central nervous system (CNS) for the treatment of PD symptoms [11]. It has been observed that LDOPA absorption is highly affected by irregular gastric emptying time, metabolism and competition with aromatic and branched-chain amino acids for absorption and transport. Also, its half-life is relatively short and it possesses a tmax of $1.4 \mathrm{~h}[9]$.

\section{Blood-brain barrier}

The BBB is a type of functional barrier that allows only the essential nutrients to enter the brain and exclude other molecules present. Its structure, although essential to keep the harmful entities out, is also a major roadblock for the pharmacological treatment of brain diseases [12]. BBB is a specialized neuroanatomical interface that is composed of unique endothelial phenotype at the brain microvascular beds which strictly enforces CNS homeostasis. The barrier functions of BBB are regulated and induced by pericytes, astrocyte, and neurons within the neurovascular unit (NVU) as shown in Figure 1 [13]. Sufficiently small solutes and

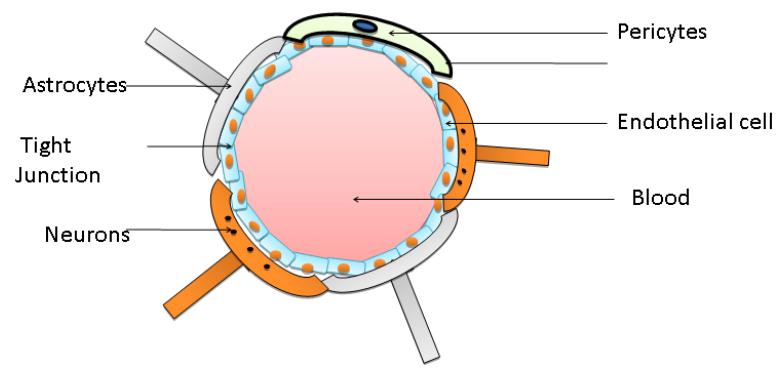

Figure 1. Structure of the Blood-brain barrier

some lipid-soluble one's effluxes out by the route provided by the BBB and also some specific transporters are present that transfer many polar substances into the brain [14]. The inter-endothelial tight junction complexes comprising occludin, 
claudins, and membrane-directed scaffolding proteins (such as zonula occludentes-1 (ZO-1)) contribute to the physical barrier nature of BBB that strictly limit the molecular/cellular influx from circulation. The P-glycoprotein (P-gp), an ATPdependent drug transport protein system is present on the luminal side of endothelial cells. This system may hinder with the accumulation of molecules in the brain, by expelling them from endothelial cells towards the bloodstream [13]. The BBB is thus the major hurdle for the passage of active molecules from the blood compartment to the brain [15]. The barrier function of brain capillaries in the BBB is primarily due to the presence of complex tight junctions, specific expression patterns of different solute carriers (SLCs) and ABC-type efflux transporters [16]. There are three types of transport mechanisms through BBB.

- Receptor-mediated transport

- Active efflux mediated transport

- Carrier mediated transport

The receptor-mediated transport includes Transferrin receptor, Insulin receptor, Insulin-like growth factor (IGH-R), Low-density lipoprotein receptor (LDL-R). Active efflux mediated transport includes $A B C$ transporter (p-glycoprotein), Glutamic acid amino acid transporter, Organic anion-transporting peptide and Carrier mediated transport includes GLUT-I transporter, Cationic amino acid transporter, Choline transporter.

\section{Measures to overcome BBB}

A number of novel brain targeting strategies employed in recent years include viral vectors, exosomes, delivery through active transporters in the BBB, brain permeability enhancer. Other noninvasive techniques to enhance brain drug uptake are microbubble-enhanced diagnostic ultrasound (MEUS) are also widely used. BBB can be overcome by using colloidal systems such as micelles, liposomes, nanoparticles that are administered through the intravenous (i.v) route. Only small and hydrophilic surface coated colloidal particles can escape opsonization, and remain in the bloodstream for relatively prolonged time periods. All these strategies employed are associated with one or more drawbacks. Alternative routes of administration such as nose to brain drug delivery could be a more potential strategy which is associated with lesser limitations. Alternative brain delivery strategies include [12]-

- Intra-Cerebro-ventricular (ICV) injection

- Intracerebral (IC) implantation
- Convection-enhanced diffusion (CED)

- Intranasal delivery

Particles that do not pass BBB

- Large size particles (>200nm)

- Highly charged particles

- Toxic substances are actively thrown out by P-glycoprotein

- Protein binding drugs cannot cross BBB because of the big size of the complex formed

Particles that can pass BBB

- Small particles less than $200 \mathrm{~nm}$ size like $\mathrm{H} 2 \mathrm{O}, \mathrm{CO} 2, \mathrm{O} 2$, etc.

- Hydrogen, a proton is highly charged, so do not permeate BBB.

- Glucose molecule (mannose, galactose, xylose, sucrose), although they are of larger sizes but are very much required by the brain so there are special facilitated transporters to transport glucose. ExampleGLUT-I [17].

- Barbiturate drugs have good permeation capability

- Many amino acids are also transported by carrier-mediated transporter

- A large number of sphingomyelins has log $(P)$ positive, hence lipophilic drug crosses the BBB membrane.

\section{Nose-to-brain drug delivery}

The nasal route is the most permeable and highly vascularized routes for drug administration, with a large surface area $(150 \mathrm{~cm} 2)$, a high total blood flow per $\mathrm{cm} 3$ and low enzyme levels in comparison to the gastrointestinal tract and liver, resulting in rapid absorption and the onset of therapeutic action [18]. Due to these reasons, nose-to-brain delivery is a simpler and direct way for brain targeting, which avoids bloodstream clearance and invasive methods. This may only work due to the unique connection that is provided by the olfactory and trigeminal nerves between the brain and external environments: following intranasal administration, drugs are exposed to the nasal mucosa, which is innervated by olfactory and trigeminal nerves [19]. Figure 2 is shown to demonstrate the possible pathways after intranasal drug delivery. Thus, the major routes of intranasal delivery are the olfactory (olfactory epithelium) and the trigeminal nerve pathways (respiratory epithelium). On intranasal administration, the administered drug molecules come in contact with the nasal mucosa, which is innervated by olfactory nerves, the trigeminal nerves and is composed of olfactory epithelium and lamina propria [12]. 
Intranasal administration is a non-invasive route that provides direct access from the nasal cavity to the brain through the olfactory and trigeminal nerves, bypassing the BBB and the pre-systemic gastrointestinal and hepatic elimination [20].

The nasal cavity is divided into four parts: nasal vestibule, nasal atrium, respiratory epithelium, and olfactory epithelium. The olfactory epithelium is formed by various cell types, and by the underlying lamina propria, which contains blood vessels, axons, glands, and connective tissue; the respiratory region instead consists of ciliated epithelial cells and mucus-secreting goblet cells [12]. Mucus or nasal secretion is present in both the two epithelium layers, in order to favor Mucociliary clearance (MCC). Mucociliary clearance regulates the residence time of the drug in nasal mucosa [19]. The olfactory epithelia are the most likely site for the direct nose-to-brain delivery: it chiefly consists of the basal cells, the sustentacular cells, and the olfactory neural cells. Basal cells are precursor cells that provide mechanical support to other cells. The olfactory neural cells or the axons are unmyelinated and interspaced between the supporting cells. They arise at the olfactory bulb in the brain and abolish at the apical surface of the olfactory epithelium. The olfactory knob protrudes out from and above the apical surface of the olfactory epithelium. The cilia are non-motile in the olfactory region with respect to the respiratory region [12]. The average diameter of olfactory axons in humans is in the range of $100-700 \mathrm{~nm}$. Therefore, theoretically, the transcellular transport of nanoparticles is possible. In the olfactory region, neurons are interspersed among supporting cells and basal cells to form the olfactory epithelium [19]. Moreover, due to the direct contact with toxins in the external environment, olfactory receptor neurons regenerate every 3-4 weeks from basal cells residing in the olfactory epithelium [12].

Another important pathway for the nose-to-brain delivery is the trigeminal nerve, which triggers the respiratory, but also the olfactory epithelium, and enters the CNS in the Pons. From the respiratory epithelium, the ophthalmic and maxillary (nasal branches) divisions of the trigeminal nerve enter the brain through anterior lacerated foramen near the pons. Transfer of substances from the nose into the brain can occur by slow intra-axonal transport (intra-neuronal), or by faster transfer along the perineural space surrounding the nerve cells into the cerebrospinal fluid (CSF), and/or into the interstitial fluid of the brain (extra-neuronal or paracellular transport) [21] Several enzymes are present in the nasal cavity such as cytochrome P450 enzyme isoforms, carboxylesterases and glutathione S-transferases which may play a role in metabolism of drugs intranasally [12].

The neuronal link between the nasal mucosa and the brain provides a unique pathway for the noninvasive delivery of therapeutic agents. Since the blood supply to the respiratory epithelium is relatively high compared to the olfactory epithelium, it is an ideal site for systemic absorption of nasally applied drugs, in order to bypass the firstpass effect- a common drawback of oral drug delivery [22].

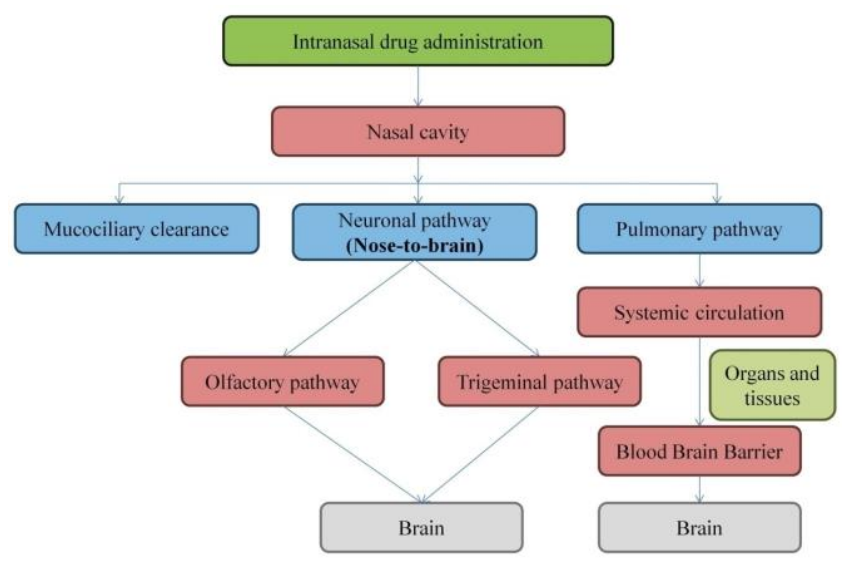

Figure 2. Various pathways followed by drug after its intranasal delivery

\section{Nanocarriers based delivery systems for Nose- to-brain drug delivery}

The nanocarriers based drug delivery systems that can be utilized for nose-to-brain drug delivery can be as polymer-based nanocarriers, lipid-based nanocarriers or polymer-lipid hybrid based nanocarriers. Among colloidal carriers, vesicular systems (liposomes, niosomes), nanoemulsions, lipid, and polymeric nanoparticles are the most promising for nasal drug delivery, especially with regard to their biocompatibility. Figure $\mathbf{3}$ represents a schematic diagram showing nanocarriers based nose-to-brain drug delivery for brain targeting. One of the key considerations for the development of nasal dosage forms is their safety and toxicological assessment of the matrix. The extended contact of formulations with nasal mucosa may lead to irritation, tissue damage, epithelial/subepithelial toxicity or ciliotoxicity, and may result in an environment suitable for microbial growth. In addition, intranasal drug formulation should not damage the primary olfactory nerves and the sense of smell. A number of lipid/polymerbased nanocarriers have been exploited in the last few decades for the successful delivery of cargos at the targeted organ i.e. brain via nose-to-brain drug delivery. A few have been reviewed in the following 
sections with their outcomes and also shown in Table 1.

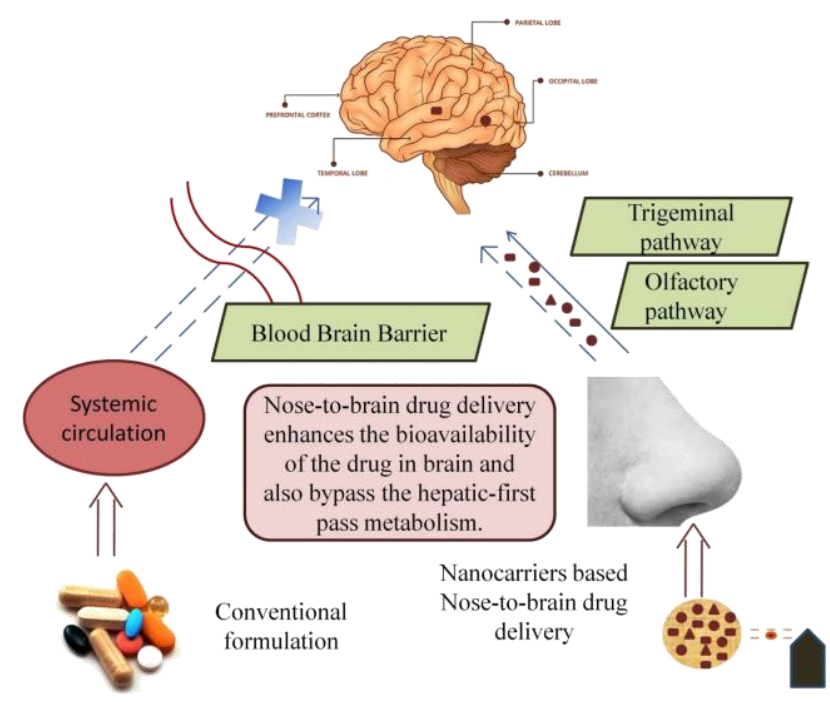

Figure 3. Schematic representation showing nanocarriers based nose-to-brain drug delivery for brain targeting

\section{Nanoemulsion/Microemulsion}

Nano metric-sized transparent or translucent dispersions of two immiscible phases with a mean droplet size between 20 to $200 \mathrm{~nm}$ are called the nanoemulsion. They are a kinetically stable isotropic system in which the immiscible liquids are mixed to form a single-phase by means of an appropriate surfactant or a mixture of a surfactant and co-surfactant in a certain ratio, known as Smix [23]. They have a higher surface area and free energy than macro emulsions and are stable against sedimentation, flocculation, and coalescence and creaming. They enhance the solubility of lipophilic drugs and improve permeation across the mucosa [24]. The preparation of nano $\neg$ emulsion with a sufficiently low particle size requires a high emulsification method [25]. that physically stable vitamin $E$ nanoemulsion encapsulating resveratrol has been developed and the result confirms that resveratrol nanoemulsion has provided relatively maximum in vitro as well as significantly high ex vivo trans-nasal mucosal flux. The higher resveratrol concentration in the brain proved the better targeting efficiency of nanoemulsion through the intranasal route. The insignificant change in zeta potential and particle size upon storage for short-term stability assures a long shelf life [18]. Authors from another study stated that Letrozole loaded nanoemulsion (LETNE) were prepared by aqueous micro-titration method. Nanoemulsion was studied for droplet size, polydispersity index (PDI), zeta potential, percentage transmittance, drug content, surface morphology. The particle size of LET-NE was established by dynamic light scattering and was later confirmed by the TEM examination. The comparative in vitro and ex-vivo studies had shown an improved therapeutic response in comparison to LET suspension. Moreover, i.n. administration of LET-NE further improved the brain uptake as compared with the i.p. route due to direct nose-tobrain delivery [29]. In one of the recent studies, Diazepam microemulsion (MEs) were prepared by the phase titration method, optimized by using BoxBehnken design. Optimized MEs, mucoadhesive microemulsion (MMEs), and Calmpose (i.v route) were evaluated for pharmacokinetic and pharmacodynamic studies. Pharmacokinetic parameters Cmax, Tmax, AUC for brain and plasma were calculated using Win Nonlin software of Version 5.2. The authors have concluded that brain targeting of diazepam mucoadhesive ME and microemulsions via nasal route is a very promising non- invasive approach superior to the intravenous route of administration with fewer side effects [30]. Lupe C. Espinoza et al. in their study revealed that Donepezil microemulsion was developed by the construction of pseudo ternary phase diagrams and characterized by dynamic light scattering and transmission electron microscopy. The lipid droplets had a spherical shape and smooth and regular surface. The microemulsion has a Newtonian flow and a viscosity value of less than 50 $\mathrm{mPa}$, which facilitates administration by intranasal sprays. anion-transporting was stable and do not present significant changes in organoleptic characteristics. In vitro release study of Donepezil indicated that the microemulsion follows a kinetic model of Hyperbola, ensuring the prolonged release of the drug, while the ex vivo permeation profile demonstrated that the highest permeation occurred during the initial $4 \mathrm{~h}$ [31].

\section{Polymeric and metallic nanoparticles}

Nanoparticles are colloidal systems with a compact structure where the therapeutic agent is either entrapped within the colloid matrix or coated on the particle surface by conjugation or adsorption [32]. Nanoparticles possess relatively high drug loading and small size and deliver the active ingredient to the specific site at a controlled and sustained rate during transportation [4]. Polymeric nanoparticles, especially those incorporating biodegradable polymers, have set the way forward for nasal drug delivery systems. The advantages of such polymeric nanoparticles are their small size, better uptake by cells, and the accumulation of drugs at the targeted sites. Significant formulation 
Table 1. A few successful examples of lipid/polymer-based nanocarriers for nose-to-brain delivery of drugs with their outcomes

\begin{tabular}{|c|c|c|c|c|}
\hline Drug & Disease & Carrier system & Outcome & Reference \\
\hline Resveratrol & Parkinson's disease & Nanoemulsion & Better targeting efficiency & {$[20]$} \\
\hline Letrozole & Status epilepticus & Nanoemulsion & $\begin{array}{l}\text { Better anticonvulsant and } \\
\text { neuroprotective action }\end{array}$ & [29] \\
\hline Risperidone & Schizopherenia & $\begin{array}{l}\text { Chitosan } \\
\text { nanoparticles }\end{array}$ & Bioefficacy enhancement & {$[34]$} \\
\hline Melatonin & Glioblastoma & nanoparticles & $\begin{array}{l}\text { Increased MLT concentrations in } \\
\text { the brain }\end{array}$ & {$[36]$} \\
\hline $\begin{array}{l}\text { Curcumin } \\
\text { cocrystals }\end{array}$ & $\begin{array}{l}\text { Neurodegenerative } \\
\text { Ailments }\end{array}$ & Micelles & $\begin{array}{l}\text { enhanced bioavailability, higher } \\
\text { brain uptake, retention time }\end{array}$ & {$[41]$} \\
\hline Doxorubicin & Glioblastoma & PVA nanogel & Site specific targeting & {$[47]$} \\
\hline $\begin{array}{l}\text { Almotriptan } \\
\text { malate }\end{array}$ & Migraine & $\begin{array}{l}\text { Solid lipid } \\
\text { nanoparticles }\end{array}$ & Higher safety profile & {$[60]$} \\
\hline Agomelatine & $\begin{array}{l}\text { Neurodegenerative } \\
\text { disorders }\end{array}$ & $\begin{array}{ll}\text { Solid } & \text { lipid } \\
\text { nanoparticles } & \end{array}$ & $\begin{array}{l}\text { Enhanced bioavailability and } \\
\text { brain drug delivery }\end{array}$ & {$[58]$} \\
\hline$\alpha$-Mangostin & Alzheimer's disease & $\begin{array}{l}\text { Transferrin } \\
\text { liposomes }\end{array}$ & $\begin{array}{l}\text { Brain-targeting ability is } \\
\text { enhanced }\end{array}$ & {$[64]$} \\
\hline Levodopa & Parkinson's disease & $\begin{array}{l}\text { Chlorotoxin- } \\
\text { modified liposomes }\end{array}$ & Better therapeutic effects & 65 \\
\hline
\end{tabular}

advantages of superior diffusion, non-toxicity, and reduced particle size are offered through the delivery of peptide drugs encapsulated in hollow nanoparticles containing chitosan (CHS) and this is produced by electrospraying. Chitosan, the deacetylated form of chitin, has been used extensively in the preparation of nanoparticles. Its biocompatibility, biodegradability, low toxicity, and mucoadhesive nature makes it ideal for the preparation of polymeric nanoparticles [33]. The mucoadhesive nature of the CHS is especially important in nasal delivery systems as the nasal cavity is subjected to ciliary clearance. Chitosan increases the residence allowing better drug diffusion across the nasal mucosa [34]. In a recent study, dopamine with borneol and lactoferrin comodified nanoparticles (Dopamine Lf-BNPs) were prepared using the double emulsion solvent evaporation method and evaluated for physicochemical and pharmaceutical properties. In vitro cytotoxicity studies indicated that treatment with dopamine Lf-BNPs has relatively low cytotoxicity. Qualitative and quantitative cellular uptake experiments demonstrated that $\mathrm{Lf}$ modification of NPs increased cellular uptake of SH-SY5Y cells and 16HBE cells, and borneol modification can promote the cellular uptake of 16HBE [35]. Mohsin Qureshi et al. in their study reported that Risperidone loaded chitosan lipid nanoparticle (RIS-CH-LNPs) were designed to enhance the bioactivity in schizophrenia via the nasal route. The optimized formulation RIS-CHLNPopt was further evaluated for its surface morphology, ex vivo permeation study, in vivo behavior study, and stability study. The developed RIS-CH-LNPs showed a nanometric size range with high drug loading and prolonged drug release. Invivo behavior studies showed that RIS-CH-LNPopt is able to show significantly greater bioefficacy as compared to RIS-SUS [intranasal (i.n), intravenous (i.v)]. Developed chitosan nanoparticles showed higher pharmacokinetic and brain/plasma ratio at all time-points as compared to RIS-SUS which is either given by intranasal or intravenous. And also nose-to-brain drug delivery was increased by developed chitosan nanoparticle as compared to the dispersion of equivalent dose [34]. Melatoninnanoparticles (MLT-NP) were prepared by the nanoprecipitation method by Junior et al. Brain and plasma pharmacokinetic profiles were analyzed. U87MG glioblastoma cells and MRC-5 non-tumor cells were used for the evaluation of cytotoxicity. MLT-NP was suitable to reach the brain after intranasal administration, allowing for increased MLT concentrations in the brain. In vivo evaluation by Fluorescence molecular tomography (FMT) analysis was performed in order to observe the possible translocation of nanoparticles to the brain. FMT images displayed rapid translocation and accumulation of nanoparticles from the nasal cavity 
to the brain. In vitro release of the drug from MLTNP was compared with free MLT diffusion through the dialysis membrane. The release of MLT from nanoparticles showed an initial burst until approximately $6 \mathrm{~h}(28.7 \%)$ and a sustained release over $48 \mathrm{~h}$ (71.2\%). The results highlight MLT-NP as a promising formulation for targeting the brain tissue and for glioblastoma chemotherapeutic protocols [36].

Jinwei Xue et al. in their work formulated gold nanoparticles with the help of Paeonia mountan roots. UV-Vis spectroscopic, FTIR, HR-TEM, EDAX and XRD analysis confirmed the synthesis of gold nanoparticles. In vivo effect of synthesized nanoparticles was confirmed by treating Parkinson's induced in mice. To estimate the potency of synthesized gold nanoparticles, behavioral, biochemical and molecular analysis was performed. The results of in vivo studies authentically confirm the synthesis of gold nanoparticles using Paeonia mountan soothes the neuroinflammation and enhances motor coordination in Parkinson induced mice.

\section{Polymeric micelles}

Polymeric micelles are self-assembled core-shell nanostructures formed in an aqueous solution consisting of amphiphilic block copolymers [37]. The hydrophobic blocks of the copolymers form the core of the micelles by hydrophobic interaction, although other interactions such as electrostatic interaction [38] and stereo complex formation [39] can also be utilized as the driving force for the core formation. The hydrophilic blocks of the copolymers form the shell of the micelles and stabilize the micellar structure [40]. Polymeric Micelles have the advantage of by-passing the Pglycoprotein (P-gP) efflux since they are transported into the cells via receptor-mediated endocytosis in distinction to the typical free drug diffusion. P-gp is a drug efflux protein that hinders the distribution of many drugs to the brain, intestine, and multidrug-resistant (MDR) tumors. However, certain drawbacks are seen in such systems such formation of aggregates with a large size, which falls outside the apparently preferred size range for drug delivery systems using nanoscale particles and also lacks stability in aqueous dispersion that may lead to phase separation. Preshita P. Desai et al. in their study states, the curcumin cocrystals were successfully developed using green scalable planetary ball mill technology, and the process was optimized and validated by the design of the experimental approach using 32 factorial designs. The developed micelles were characterized using DSC,
XRD, and FTIR analysis. The cocrystal micelles exhibited spherical geometry with a globule size of $28.79 \pm 0.86 \mathrm{~nm}$. Significantly low IC50 concentration was exhibited by curcumin cocrystal and cocrystal micelles for antioxidant activity when compared to curcumin and thus this confirms the superior antioxidant performance of it. In vivo studies revealed a 1.7-fold increase in the bioavailability of curcumin cocrystal micelles with delayed clearance. The studies confirmed enhanced bioavailability, higher brain uptake, retention, and delayed clearance with curcumin cocrystal micellar nanocarriers [41]. In another study, an evaluation was done for the nose-to-brain delivery of siRNA that targets TNF- $\alpha$ (siTNF- $\alpha$ ) which is in conjugation with PEG-PCL-Tat to investigate its therapeutic effects on a transient middle cerebral artery occlusion (t-MCAO) rat model of cerebral ischemia-reperfusion injury. The untreated group showed the infarcted area over a wide range, whereas on the other hand after intranasal administration of siTNF- $\alpha$ with PEG-PCL-Tat micelles showed shrinkage of the infarcted area. Thus, the results indicated that nose-to-brain delivery of siTNF- $\alpha$ conjugated with PEG-PCL-Tat micelles alleviated the symptoms of cerebral ischemia-reperfusion injury [42].

\section{Nanogels}

The nanogels are mainly nanosized hydrogel and can also be defined as chemically or physically crosslinked three-dimensional networks of polymers that swell in water or aqueous fluid $[43,44]$. Enhanced biocompatibility is due to the higher water content of hydrogel and promotes the diffusion of the drug from the swollen network of the polymer [45]. As compared to neutral nanogels, the cationic nanogels boost the internalization of nanocarrier in the cells [46]. Chen et al. in their study concluded that to target the human glioblastoma or tumor cell, they have developed doxorubicin-loaded pH-responsive PVA nanogel. The surface of the nanogel was modified with cycloRGDpeptide, in the normal physiological conditions the carrier system remains inactive and becomes active when reaches to the tumor site due to change in $\mathrm{pH}$. The adverse effects are also reduced by surface modification [47]. Warren et al. in their study have developed biodegradable cationic nanogel so as to deliver triphosphorylated nucleoside reverse transcriptase inhibitor into the brain to treat HIV. So as to reduce the peripheral side effects and neurotoxicity, the nanogel was made of cholesterol- $\varepsilon$-polylysine [48]. The results were found to be favourable as expected.

\section{Dendrimers}


Dendrimers are monodispersed, highly branched and symmetric polymeric macromolecules $[49,50]$. To improve the drug loading ability the periphery is closely packed and the reactive ends of surface allow multifunctionality. The nano-sized dendrimers are the best-suited carrier system for brain targeting [46]. Lu et al. have synthesized arsenic trioxide loaded RGDPEG-modified polyamidoamine (PAMAM) for targeting brain glioblastoma cells. To reduce the cytotoxicity the surface was modified with PEG. The therapeutic efficiency and pharmacokinetic profile of the drug were found to be increased by prolonged drug release [51]. Gothwal et al. have prepared rivastigmine loaded lactoferrin modified polyamidoamine dendrimers to boost the memory function of the brain and to treat the neurodegenerative disorders. The dendrimer enhanced bioavailability and improved locomotor activity and memory [52].

\section{Lipid nanoparticles/ nano lipid carriers (NLC)}

Nanoparticles can improve nose-to-brain drug delivery due to the presence of P-gp efflux proteins that protect the encapsulated drug from extracellular transport and biological or/and chemical degradation. Moreover, retention of bioadhesive nanoparticles on the mucosal surface of the nasal cavity, as well as their ability of transiently opening of the tight junctions of the mucosal epithelium (because of surfactants used in the formulation) should contribute to enhanced brain drug delivery [53]. The Transmucosal transport of nanoparticles from nose-to-brain may take place through olfactory neurons via various endocytic pathways of neuronal cells [12]. Lipid nanoparticles (LN) are particles in the $50-1000 \mathrm{~nm}$ range, wherein the matrix is made of biocompatible solid lipids or mixtures of solid and liquid lipids. So far, when compared to other colloidal systems, LN has been described as superior carriers, with various advantages [54]. The LNs are further divided into 3 subtypes- solid lipid nanoparticles (SLN), nanostructured lipd carriers (NLC) and lipid nano-emulsions (LNE). Confocal microscopy revealed that fluorescent-labeled polystyrene nanoparticles of diameter in the range of 20-200 nm was involved with clathrin-coated pits whereas nanoparticles of size in the range of 200$1000 \mathrm{~nm}$ were found to be involved with caveolaemediated endocytosis [55]. Recently attempted experimental approaches with nose-to-brain delivery for glioma treatment states, this administration route is problematic for cytotoxic drugs that can cause local damage in the nose cavity after administration [12]. In a recent study Teriflunomide-loaded NLC (TFM-NLC) nanoparticles were prepared by melt emulsification ultrasonication method using biodegradable and biocompatible polymers for the treatment of multiple sclerosis. Particle sizes, entrapment efficiency (\%), in vitro and ex vivo permeation was evaluated for the optimized NLC formulation. The safety and efficacy of optimized formulations were demonstrated using pharmacodynamic, sub-acute toxicity and hepatotoxicity data. The rapid remyelination in cuprizone treated animals and decreases the number of entries in the open compartment of EPM was produced by the TFMMNLC (i.n.) formulation. Hence, the nose-to-brain delivery of TFM-MNLC can be considered as an effective and safe delivery for brain disorders [56]. Brijesh Shah et al. in their study have applied the $\mathrm{QbD}$ approach was for the development of hydrophilic drug (RHT) loaded SLN as a novel concept. RHT SLN was prepared by homogenization and ultrasonication method. High \%EE in SLN indicated higher incorporation of RHT. RHT SLN being lipidic in nature showed higher drug diffusion in comparison to drug solution having the crystalline form of the drug. This was further consolidated by DSC and XRD data which showed that RHT has lost its characteristic crystalline nature. RHT SLN did not show any nasociliary damage and/or cell necrosis indicating its safety for nasal administration [57].

\section{Solid lipid nanoparticles (SLN)}

These are colloidal particles having a size ranging from 1 to $1,000 \mathrm{~nm}$. They are composed of lipids with melting points higher than the room temperature [58]. They are preferred over other colloidal carriers because of their lipophilic nature and other versatile properties like high drug payload, controlled release, drug targeting and feasibility to incorporate both hydrophilic and lipophilic drugs make Solid Lipid Nanoparticles an efficacious carrier system for a wide range of therapeutics facing challenges in the area of brain targeted delivery system [59]. As compared to the NLCs, SLN has low drug loading capacity due to high crystallinity and rapid drug expulsion during long term storage. The SLNs were prepared by some researchers using $\mathrm{w} / \mathrm{o} / \mathrm{w}$ double emulsion solvent evaporation technique. The pharmacokinetics and bio-distribution evaluation revealed that intranasal in-situ gel-based formulae; NF (SLNs based) is a good candidate for Almotriptan malate nose-to-brain targeting. Biomarkers' evaluation and histopathological examination results indicated the higher safety profile of NF (in-situ gel-based SLNs) for nasal administration. Toxicological results confirmed the safety of NF for nasal administration [60]. Ahmed M 
Fatouh et al. in their study concluded that they have selected solid lipid nanoparticles to increase the permeability of agomelatine across the BBB. The pharmacokinetic study of the optimized SLN revealed the increase in the plasma peak concentration, the AUC and absolute bioavailability. Better brain targeting was observed by the intranasal route as compared with the intravenous route as the optimized nanoparticles gave a better drug-targeting efficiency. Due to avoidance of the first-pass metabolism, the prepared formulation effectively enhanced both the absolute bioavailability and the brain delivery, the favored BBB uptake of the SLNs and the direct transport from the nose to the brain.[58].

\section{Emulsomes}

Emulsomes (lipid-based nanoparticles) are a nanocarrier composed of lipid cores either in the solid or liquid crystalline state, with a covering of phospholipid bilayer. Emulsomes comprises of the characteristics of both lipid spheres as well as liposomes. The higher extent of lipophilic drugs can be encapsulated due to the presence of lipid spheres. Particles formed in emulsomes are similar to those of micelles, hence bioavailability and solubility increase [61]. In a study by Ghada M. ElZaafarany et al. evaluated the pharmacokinetic studies in rats for the transport of Oxcarbazepine to the systemic circulation after nasal administration with higher uptake in the brain tissue in case of Oxcarbazepine loaded emulsomes. Mild vascular congestion and moderate inflammatory changes have been observed by histopathological study. In the same study, the PLGA-PEG-PLGA triblock copolymer was mixed with emulsomes to form thermoresponsive gels for the nasal delivery of Oxcarbazepine. The Oxcarbazepine-emulsome thermogel dramatically increased the systemic Oxcarbazepine absorption but did not improve the Oxcarbazepine brain concentration in terms of Cmax and AUC as compared to the Oxcarbazepine bearing emulsomes alone. However, the emulsomal thermogel extended the presence of Oxcarbazepine in the brain to up to $48 \mathrm{~h}$, which could offer better control of seizures for a longer time period [61].

\section{Liposomes}

Liposomes are biodegradable colloids that can be employed to transfer a wide range of hydrophobic and hydrophilic pharmaceuticals, such as small molecules, peptides, proteins, and RNAs, without changing their function and protecting them against degradation [4]. The phospholipid bilayer structure of liposomes made it easier to penetrate
BBB and helps therapeutic molecules to enter the brain. Liposomes have low toxicity and the ability to deliver both hydrophilic and lipophilic compounds [62]. However, there are several limitations of liposomes, including fast systemic elimination, quick metabolic degradation of the phospholipids, stability issue after extended storage, inability to provide sustained release of drugs and moderately efficient for the entrapment of lipophilic compounds [63]. Liposomes are highly lipophilic colloidal carriers that can encapsulate hydrophilic agents (proteins of interest) in the aqueous core and protect the payload from degradation. The advantages of liposomes like high stability, better pharmacokinetics and biodistribution profile, drug release kinetics have made them one of the most successful delivery systems. In one research, these liposomes have improved transmucosal diffusion and also may be engineered to provide a sustained release by incorporating them into a thermosensitive, mucoadherent gel carrier such as Pluronic F-127, chitosan, etc. The liposomes-in-gel (LiG) formulation offers several advantages like tissue compatibility, ease of application, stability and sustained release of the payload over time. Nowadays, plenty of modifications have been made in the liposomal surface to improve its brain targeting ability. With the help of some specific ligands (glucose, lactoferrin, transferrin, specific peptides), the liposome can efficiently cross the BBB and deliver the drug to the particular site [4]. Different kinds of modified liposomes are becoming the choice of carriers for the formulation scientists such as Transferrin modified liposomes, Glutathione modified liposomes, Cationic liposomes, PEG-modified liposomes, Multifunctional liposomes, etc. Transferrin modified liposomes of $\alpha$-Mangostin were formulated for the treatment of $A D$ by a group of researchers. The efficacy of $\alpha$-Mangostin was limited by the poor penetration of the drug through the BBB. Transferrin (Tf) was used as a targeting ligand to modify the liposome to achieve the desired penetrating effects. Qualitative and quantitative experiments proved that the Tf-liposome could promote brain-targeting ability [64]. Xiang $Y$ et al. have formulated Chlorotoxin-modified stealth liposomes (CITx -LS) of levodopa for the treatment of PD. The in vitro and in vivo results showed good correlation with each other and both proved the hypothesis that CITx -LS may be a potential active targeting system to promote the drug delivery into the brain to achieve a better PD therapy [65]. Lazar et al. in a study prepared Curcumin conjugated liposome for the treatment of $A D$. Curcumin is a fluorescent molecule with a high affinity for the $A \beta$ peptide but its low solubility limits its clinical use. Demonstrated strong labeling of $A \beta$ deposits both in human tissue and in mice, and in vitro 
Table 2. A few examples of patents filed related to nasal drug delivery for brain targeting

\begin{tabular}{|c|c|c|}
\hline Patent number & Patent title & Summary of invention \\
\hline AU2014223679B2 & $\begin{array}{l}\text { Treatment of central nervous system } \\
\text { disorders by intranasal administration of } \\
\text { immunoglobulin } G\end{array}$ & $\begin{array}{l}\text { methods and compositions for treating Alzheimer's } \\
\text { disease by delivering immunoglobulin } G \text { via } \\
\text { intranasal administration }\end{array}$ \\
\hline CA2970917A1 & $\begin{array}{l}\text { Methods of treating inflammatory } \\
\text { disorders and global inflammation with } \\
\text { compositions comprising phospholipid } \\
\text { nanoparticle encapsulations of NSAIDs }\end{array}$ & $\begin{array}{l}\text { Phospholidip nanoparticles encapsulate NSAIDs } \\
\text { yielding an increase in NSAID transport across } \\
\text { hydrophobic mucosa; increase the bioavailability } \\
\text { of the NSAID, decrease the dose of NSAIDs as } \\
\text { compared to pills. }\end{array}$ \\
\hline WO2017041037A1 & $\begin{array}{l}\text { Disruption of the interaction between } \\
\text { amyloid-beta peptide and dietary lipids }\end{array}$ & $\begin{array}{l}\text { The invention relates to methods and compositions } \\
\text { for intranasal administration of lipids containing } \\
\text { fatty acid acyl chains of dietary lipids for } \\
\text { promoting central nervous system health and/or } \\
\text { prevention of neurodegenerative disorders }\end{array}$ \\
\hline US20180338910A1 & $\begin{array}{l}\text { Intranasal administration of glutamate } \\
\text { carboxypeptidase (GCP-II) inhibitors }\end{array}$ & $\begin{array}{l}\text { GCP-II has been demonstrated to be efficacious in } \\
\text { multiple preclinical models wherein excess } \\
\text { glutamate transmission is implicated including } \\
\text { traumatic spinal cord and brain injury, stroke, } \\
\text { neuropathic and inflammatory pain, ALS. GCP-II } \\
\text { has demonstrated long term memory-enhancing } \\
\text { effects in the animal study. }\end{array}$ \\
\hline WO2019053625A1 & $\begin{array}{l}\text { System and Method for Automated } \\
\text { Personalized Brain Modulation with } \\
\text { Photobiomodulation }\end{array}$ & $\begin{array}{l}\text { The present invention provides a system and } \\
\text { method whereby the PBM device works in } \\
\text { combination with a diagnostic tool to provide } \\
\text { enhanced treatment of abnormal brain function } \\
\text { intelligently, automatically, and unrestricted by } \\
\text { geographical distances. }\end{array}$ \\
\hline US20170239209A1 & $\begin{array}{l}\text { Intranasal Compositions For Treatment of } \\
\text { Neurological And Neurodegenerative } \\
\text { Diseases And Disorders }\end{array}$ & $\begin{array}{l}\text { The present invention is directed to sustained, } \\
\text { enhanced delivery of pharmaceutical agents } \\
\text { across the nasal mucosa for systemic delivery of } \\
\text { anti-Alzheimer's drug }\end{array}$ \\
\hline
\end{tabular}

downregulation of amyloid peptide secretion and prevention of $A \beta$-induced toxicity [66].

\section{Recent advances, future prospective, and toxicity concerns}

Besides the above examples, a number of patents have also been filed related to nasal drug delivery for brain targeting. A few examples of patents are given in Table 2. In addition to these many formulators have successfully launched similar products in the market also for commercial use. Marketed formulation IMITREX (sumatriptan) Nasal Spray is a product of GlaxoSmithKline contains sumatriptan as active ingredient, a selective 5hydroxytryptamine 1 receptor subtype agonist, used in the treatment of migraine. Several human trials have been conducted via nose-to-brain delivery for various drugs like Diphenhydramine hydrochloride shows antitussive, antihistaminic property [67], Doxylamine succinate shows antidepressant activity [68], ergotamine tartrate can be used to treat migraine [69] and propranolol hydrochloride can be used in the treatment of hypertension and angina pectoris [70]. Nanocarriers are considered a powerful tool to penetrate the BBB but there are many concerns that must be looked after. First, as the specific distribution is not clear after the absorption of massive nanocarriers into the brain, this may cause risk in such an influential organ [2]. The metabolism of nanocarriers is an important factor. Most of the nanocarriers are composed of inorganic materials, like gold nanoparticles, iron nanoparticles, cerium oxide nanoparticles, molybdenum nanoparticles and silica nanoparticles which cannot be easily metabolized, which may result in accumulation in the brain. Such nanocarriers cause neurodegeneration by promoting mitochondrial dysfunction, redox imbalance and apoptosis, autophagy and impaired lysosomal activity [71-74]. Biodegradable nanoparticles also showed neurotoxicity in various researches. For example, after polysorbate 80-modified chitosan nanoparticles injecting into the body, the body weight was found to remarkably decrease in a dose-dependent manner for seven days, also causing apoptosis, necrosis of neurons [75]. Moreover, chitosan nanoparticles at a size of 200 $\mathrm{nm}$ cause impairments, including a bent spine, pericardial edema, and an opaque yolk in the embryos of zebrafish $[76,77]$.

\section{Conclusion}

The BBB and the blood-cerebrospinal fluid barrier 
are major obstacles in drug delivery to the brain for the management of several neurodegenerative disorders. Intranasal delivery has shown to circumvent BBB and deliver the drugs into the CNS at a higher rate and extent than other conventional routes. Nose-to-brain delivery of drugs via lipid/polymer-based nanocarriers is established as an efficient approach to delivering drugs directly to the brain. A number of in vitro, ex vivo and preclinical studies have suggested the success of the present approach. However, the real potential of this treatment modality could be assessed and warranted through highly extensive clinical trials to address the issue of safety and long-term toxicity of the developed nanocarriers for nose-to-brain drug delivery.

\section{Acknowledgement}

We acknowledge the Department of Pharmaceutics, ISF College of Pharmacy, Moga, India for providing resources and funding for the research

\section{Conflict of interest}

The authors confirm that this article content has no conflicts of interest.

\section{References}

[1] Choonara YE, Pillay V, Du Toit LC, Modi G, Naidoo D, Ndesendo VMK, et al. Trends in the Molecular Pathogenesis and Clinical Therapeutics of Common Neurodegenerative Disorders 2009; 10: 2510-57.

[2] Nakamura T, Prikhodko OA, Pirie E, Nagar S, Akhtar MW, Oh C-K, et al. Aberrant protein Snitrosylation contributes to the pathophysiology of neurodegenerative diseases 2015; 84: 99-108.

[3] Agrawal M, Saraf S, Saraf S, Antimisiaris SG, Chougule MB, Shoyele SA, et al. Nose-tobrain drug delivery: An update on clinical challenges and progress towards approval of anti-Alzheimer drugs 2018; 281: 139-77.

[4] Niu X, Chen J, Gao J. Nanocarriers as a powerful vehicle to overcome blood-brain barrier in treating neurodegenerative diseases: Focus on recent advances 2019; 14: 480-96.
[5] Dyer AM, Smith A. Riluzole $5 \mathrm{mg} / \mathrm{mL}$ oral suspension: for optimized drug delivery in amyotrophic lateral sclerosis 2017; 11: 59.

[6] Bryan MR, O'Brien MT, Nordham KD, Rose DI, Foshage AM, Joshi $P$, et al. Acute manganese treatment restores defective autophagic cargo loading in Huntington's Disease cell lines 2019.

[7] Tonda-Turo C, Origlia N, Mattu C, Accorroni A, Chiono V. Current Limitations in the Treatment of Parkinson's and Alzheimer's Diseases: State-of-the-Art and Future Perspective of Polymeric Carriers 2018; 25: 5755-5771.

[8] Cattaneo C, Jost WH, Bonizzoni E. Long-Term Efficacy of Safinamide on Symptoms Severity and Quality of Life in Fluctuating Parkinson's Disease Patients 2019: 1-9.

[9] Seeberger LC, Hauser RA. Optimizing bioavailability in the treatment of Parkinson's disease 2007; 53: 791-800.

[10] Hoy SM. Levodopa/Carbidopa Enteral Suspension: A Review in Advanced Parkinson's Disease 2019: 1-10.

[11] Ngwuluka N, Pillay V, Du Toit LC, Ndesendo V, Choonara Y, Modi G, et al. Levodopa delivery systems: advancements in delivery of the gold standard 2010; 7: 203-224.

[12] Battaglia L, Panciani PP, Muntoni E, Capucchio MT, Biasibetti E, De Bonis $P$, et al. Lipid nanoparticles for intranasal administration: application to nose-to-brain delivery 2018; 15: 369-378.

[13] Sajja RK, Rahman S, Cucullo L. Drugs of abuse and blood-brain barrier endothelial dysfunction: $A$ focus on the role of oxidative stress 2016; 36: 539-554.

[14] Hladky SB, Barrand MA. Elimination of substances from the brain parenchyma: efflux via perivascular pathways and via the blood-brain barrier 2018; 15: 30.

[15] Garcia-Garcia E, Andrieux K, Gil S, Couvreur P. Colloidal carriers and blood-brain barrier (BBB) translocation: a way to deliver drugs to the brain? 2005; 298: 274-292. 
[16] Helms HC, Abbott NJ, Burek M, Cecchelli R, Couraud P-O, Deli MA, et al. In vitro models of the blood-brain barrier: an overview of commonly used brain endothelial cell culture models and guidelines for their use 2016; 36: 862-890.

[17] Hladky SB, Barrand MA. Fluid and ion transfer across the blood-brain and bloodcerebrospinal fluid barriers; a comparative account of mechanisms and roles 2016; 13: 19.

[18] Pangeni R, Sharma S, Mustafa G, Ali J, Baboota S. Vitamin E loaded resveratrol nanoemulsion for brain targeting for the treatment of Parkinson's disease by reducing oxidative stress 2014; 25: 485102.

[19] Mistry A, Stolnik S, Illum L. Nanoparticles for direct nose-to-brain delivery of drugs 2009; 379: 146-157.

[20] Lochhead JJ, Thorne RG. Intranasal delivery of biologics to the central nervous system 2012; 64: 614-628.

[21] Djupesland PG, Messina JC, Mahmoud RA. The nasal approach to delivering treatment for brain diseases: an anatomic, physiologic, and delivery technology overview 2014; 5: 709-733.

[22] Sood S, Jain K, Gowthamarajan K. Intranasal therapeutic strategies for management of Alzheimer's disease 2014; 22: 279-294.

[23] Campos FF, Campmany ACC, Delgado GR, Serrano OL, Naveros BC. Development and characterization of a novel nystatin-loaded nanoemulsion for the buccal treatment of candidosis: Ultrastructural effects and release studies 2012; 101: 3739-3752.

[24] Ganta S, Devalapally H, Amiji M. Curcumin enhances oral bioavailability and anti-tumor therapeutic efficacy of paclitaxel upon administration in nanoemulsion formulation 2010; 99: 4630-4641.

[25] Koroleva MY, Yurtov EV. Nanoemulsions: the properties, methods of preparation and promising applications 2012; 81: 21.

[26] Ferreira P, Noronha L, Teixeira R, Vieira Í, Borba-Santos L, Viçosa $A$, et al. Investigation of a microemulsion containing clotrimazole and itraconazole for transdermal delivery for the treatment of sporotrichosis 2019.

[27] Singh $M$, Kanoujia J, Parashar $P$, Arya $M$, Tripathi CB, Sinha VR, et al. Assessment of improved buccal permeation and bioavailability of felodipine microemulsionbased cross-linked polycarbophil gel 2018; 8: 591-601.

[28] Ramreddy S, Janapareddi K. Brain targeting of chitosan-based diazepam mucoadhesive microemulsions via nasal route: formulation optimization, characterization, pharmacokinetic and pharmacodynamic evaluation 2019; 45: 147-158.

[29] Bhatnagar AS. The discovery and mechanism of action of letrozole 2007; 105: 7-17.

[30] Ramreddy S, Janapareddi K. Brain targeting of chitosan-based diazepam mucoadhesive microemulsions via nasal route: formulation optimization, characterization, pharmacokinetic and pharmacodynamic evaluation 2019; 45: 147-158.

[31] Espinoza LC, Vacacela M, Clares B, Garcia ML, Fabrega M-J, Calpena AC. Development of a Nasal Donepezil-loaded Microemulsion for the Treatment of Alzheimer's Disease: in vitro and ex vivo Characterization 2018; 17 : 43-53.

[32] Wong $\mathrm{HL}$, Wu $\mathrm{XY}$, Bendayan $\mathrm{R}$. Nanotechnological advances for the delivery of CNS therapeutics 2012; 64: 686-700.

[33] Raj R, Wairkar S, Sridhar V, Gaud R. Pramipexoledihydrochloride loaded chitosan nanoparticles for nose to brain delivery: Development, characterization and in vivo anti-Parkinson activity 2018; 109: 2735.

[34] Qureshi M, Aqil Mohd, Imam SS, Ahad A, Sultana Y. Formulation and Evaluation of Neuroactive Drug Loaded Chitosan Nanoparticle for Nose to Brain Delivery: Invitro Characterization and In-vivo Behavior Study 2018; 16: 123-35.

[35] Tang S, Wang A, Yan X, Chu L, Yang X, Song $Y$, et al. Brain-targeted intranasal delivery of dopamine with borneol and lactoferrin co- 
modified nanoparticles for treating Parkinson's disease 2019; 26: 700-707.

[36] de Oliveira Junior ER, Nascimento $T L$, Salomão MA, da Silva ACG, Valadares MC, Lima EM. Increased Nose-to-Brain Delivery of Melatonin Mediated by Polycaprolactone Nanoparticles for the Treatment of Glioblastoma 2019; 36: 131.

[37] Riess G. Micellization of block copolymers 2003; 28: 1107-70.

[38] Harada A. Chain Length Recognition: CoreShell Supramolecular Assembly from Oppositely Charged Block Copolymers 1999; 283: 65-67.

[39] Kang N, Perron M-Ė, Prud'homme RE, Zhang Y, Gaucher G, Leroux J-C. Stereocomplex Block Copolymer Micelles: Core-Shell Nanostructures with Enhanced Stability 2005; 5: 315-19.

[40] Rijcken CJF, Soga O, Hennink WE, Nostrum CF van. Triggered destabilisation of polymeric micelles and vesicles by changing polymers polarity: An attractive tool for drug delivery 2007; 120: 131-48.

[41] Desai PP, Patravale VB. Curcumin Cocrystal Micelles-Multifunctional Nanocomposites for Management of Neurodegenerative Ailments 2018; 107: 1143-56.

[42] Kanazawa $T$, Kurano $T$, Ibaraki $H$, Takashima Y, Suzuki T, Seta Y. Therapeutic Effects in a Transient Middle Cerebral Artery Occlusion Rat Model by Nose-To-Brain Delivery of AntiTNF-Alpha siRNA with Cell-Penetrating Peptide-Modified Polymer Micelles 2019; 11: 478.

[43] Li X, Tsibouklis J, Weng T, Zhang B, Yin G, Feng $G$, et al. Nano carriers for drug transport across the blood-brain barrier 2017; 25: 17-28.

[44] Alexander A, Ajazuddin, Khan J, Saraf S, Saraf S. Polyethylene glycol (PEG)-Poly(Nisopropylacrylamide) (PNIPAAm) based thermosensitive injectable hydrogels for biomedical applications 2014; 88: 575-85.

[45] Weng T, Guo J, Li X, Cui Y, Zhang B, Mikhalovsky SV, et al. Synthesis,
Chloramphenicol Uptake, and In Vitro Release of Poly(AMPS-TEA-Co-AAm) Gels with Affinity for Both Water and Alcohols 2014; 63: 73-79.

[46] Alexander A, Agrawal M, Uddin A, Siddique $S$, Shehata AM, Shaker MA, et al. Recent expansions of novel strategies towards the drug targeting into the brain 2019; Volume 14: 5895-5909.

[47] Chen W, Zou Y, Zhong Z, Haag R. Cyclo(RGD)-Decorated ReductionResponsive Nanogels Mediate Targeted Chemotherapy of Integrin Overexpressing Human Glioblastoma In vivo 2017; 13: 1601997.

[48] Warren G, Makarov E, Lu Y, Senanayake T, Rivera K, Gorantla S, et al. Amphiphilic Cationic Nanogels as Brain-Targeted Carriers for Activated Nucleoside Reverse Transcriptase Inhibitors 2015; 10: 88-101.

[49] He H, Li Y, Jia X-R, Du J, Ying X, Lu W-L, et al. PEGylated Poly(amidoamine) dendrimerbased dual-targeting carrier for treating brain tumors 2011; 32: 478-87.

[50] Yan H, Wang J, Yi P, Lei H, Zhan C, Xie C, et al. Imaging brain tumor by dendrimer-based optical/paramagnetic nanoprobe across the blood-brain barrier 2011; 47: 8130-32.

[51] Lu $Y$, Han S, Zheng $H$, Ma R, Ping $Y$, Zou J, et al. A novel RGDyC/PEG co-modified PAMAM dendrimer-loaded arsenic trioxide of glioma targeting delivery system 2018; Volume 13: 5937-52.

[52] Gothwal A, Nakhate KT, Alexander A, Ajazuddin, Gupta U. Boosted Memory and Improved Brain Bioavailability of Rivastigmine: Targeting Effort to the Brain Using Covalently Tethered Lower Generation PAMAM Dendrimers with Lactoferrin 2018; 15: 4538-49.

[53] Kulkarni AD, Vanjari $Y H$, Sancheti KH, Belgamwar VS, Surana SJ, Pardeshi CV. Nanotechnology-mediated nose to brain drug delivery for Parkinson's disease: a mini review 2015; 23: 775-88.

[54] Mehnert W, Mäder K. Solid lipid nanoparticles: Production, characterization and applications 2012; 64: 83-101. 
[55] Rejman J, Oberle V, Zuhorn IS, Hoekstra D. Size-dependent internalization of particles via the pathways of clathrin- and caveolaemediated endocytosis 2004; 377: 159-69.

[56] Gadhave DG, Kokare CR. Nanostructured lipid carriers engineered for intranasal delivery of teriflunomide in multiple sclerosis: optimization and in vivo studies 2019; 45: 839-51.

[57] Shah B, Khunt D, Bhatt H, Misra M, Padh H. Application of quality by design approach for intranasal delivery of rivastigmine loaded solid lipid nanoparticles: Effect on formulation and characterization parameters 2015; 78: 54-66.

[58] Fatouh A, Elshafeey A, Abdelbary A. Intranasal agomelatine solid lipid nanoparticles to enhance brain delivery: formulation, optimization and in vivo pharmacokinetics 2017; Volume 11: 181525.

[59] Patel S, Chavhan S, Soni H, Babbar AK, Mathur R, Mishra AK, et al. Brain targeting of risperidone-loaded solid lipid nanoparticles by intranasal route 2011; 19 : 468-74.

[60] Youssef NAHA, Kassem AA, Farid RM, Ismail FA, EL-Massik MAE, Boraie NA. A novel nasal almotriptan loaded solid lipid nanoparticles in mucoadhesive in situ gel formulation for brain targeting: Preparation, characterization and in vivo evaluation 2018; 548: 609-24.

[61] El-Zaafarany G, Soliman M, Mansour S, Cespi M, Palmieri G, Illum $L$, et al. A Tailored Thermosensitive PLGA-PEGPLGA/Emulsomes Composite for Enhanced Oxcarbazepine Brain Delivery via the Nasal Route 2018; 10: 217.

[62] Costantino L, Tosi G, Ruozi B, Bondioli L, Vandelli MA, Forni F. Chapter 3 - Colloidal systems for CNS drug delivery. In: Sharma HS, Ed. Progress in Brain Research., vol. 180; Elsevier 2009; pp. 35-69.

[63] Wong $\mathrm{HL}$, $\mathrm{Wu} X Y$, Bendayan $\mathrm{R}$. Nanotechnological advances for the delivery of CNS therapeutics 2012; 64: 686700 .
[64] Chen Z-L, Huang M, Wang X-R, Fu J, Han M, Shen $Y-Q$, et al. Transferrin-modified liposome promotes $\alpha$-mangostin to penetrate the blood-brain barrier 2016; 12 : 421-30.

[65] Xiang Y, Wu Q, Liang L, Wang X, Wang J, Zhang $X$, et al. Chlorotoxin-modified stealth liposomes encapsulating levodopa for the targeting delivery against the Parkinson's disease in the MPTP-induced mice model 2012; 20: 67-75.

[66] Lazar AN, Mourtas S, Youssef I, Parizot C, Dauphin A, Delatour B, et al. Curcuminconjugated nanoliposomes with high affinity for $A \beta$ deposits: Possible applications to Alzheimer disease 2013; 9: 712-21.

[67] Gaffey MJ, Gwaltney JM, Sastre A, Dressler WE, Sorrentino JV, Hayden FG. Intranasally and Oraliy Administered Antihistamine Treatment of Experimental Rhinovirus Colds 1987; 136: 556-60.

[68] Romeo VD, deMeireles JC, Gries WJ, Xia WJ, Sileno AP, Pimplaskar HK, et al. Optimization of systemic nasal drug delivery with pharmaceutical excipients. 1998; 29: 117-33.

[69] Aellig WH, Rosenthaler J. Venoconstrictor effects of dihydroergotamine after intranasal and intramuscular administration 1986; 30: 581-84.

[70] Hussain A, Hirai S, Bawarshi R. Nasal absorption of propranolol from different dosage forms by rats and dogs 1980; 69: 1411-13.

[71] Wong $\mathrm{HL}, \mathrm{Wu} X Y$, Bendayan $\mathrm{R}$. Nanotechnological advances for the delivery of CNS therapeutics 2012; 64: 686700.

[72] Migliore L, Uboldi C, Di Bucchianico S, Coppedè F. Nanomaterials and neurodegeneration: Nanomaterials and Neurodegeneration 2015; 56: 149-70.

[73] Valdiglesias V, Kiliç G, Costa C, FernándezBertólez N, Pásaro E, Teixeira JP, et al. Effects of iron oxide nanoparticles: Cytotoxicity, genotoxicity, developmental 
toxicity, and neurotoxicity 2015; 56: 12548.

[74] Gurr J-R, Wang ASS, Chen C-H, Jan K-Y. Ultrafine titanium dioxide particles in the absence of photoactivation can induce oxidative damage to human bronchial epithelial cells 2005; 213: 66-73.

[75] Yuan Z-Y, Hu Y-L, Gao J-O. Brain Localization and Neurotoxicity Evaluation of Polysorbate 80-Modified Chitosan Nanoparticles in Rats 2015; 10: e0134722.

[76] Gao J-Q, Hu, Wang, Han. Toxicity evaluation of biodegradable chitosan nanoparticles using a zebrafish embryo model 2011: 3351.

[77] Manufactured Nanomaterials (Fullerenes, C60) Induce Oxidative Stress in the Brain of Juvenile Largemouth Bass | Environmental Health Perspectives | Vol. 112, No. 10 [homepage on the Internet]. n.d. [cited 2019 Nov 11] Available from: (https://ehp.niehs.nih.gov/doi/full/10.1289 /ehp.7021). 